\title{
Ustilago cynodontis
}

National Cancer Institute

\section{Source}

National Cancer Institute. Ustilago cynodontis. NCI Thesaurus. Code C87522.

A species of smut fungi in the phylum Basidiomycota. This species causes smut in various grasses. 